\title{
Transcriptome profiling of cancer and normal tissues from cervical squamous cancer patients by deep sequencing
}

\author{
WANSONG LIN ${ }^{1,2}$, MEI FENG $^{3}$, XIUHUA LI ${ }^{3}$, PEILIN ZHONG $^{3}$, AIHUA GUO $^{3}$, GUILIN CHEN $^{3}$, \\ QIN XU ${ }^{3}$ and YUNBIN YE ${ }^{1,2}$ \\ ${ }^{1}$ Laboratory of Immuno-Oncology, ${ }^{2}$ Fujian Provincial Key Laboratory of Translational Cancer Medicine and ${ }^{3}$ Department of \\ Gynecologic Oncology, Fujian Cancer Hospital and Fujian Medical University Cancer Hospital, \\ Fuzhou, Fujian 350014, P.R. China
}

Received May 2, 2016; Accepted April 4, 2017

DOI: $10.3892 / \mathrm{mmr} .2017 .6855$

\begin{abstract}
Cervical cancer is the fourth leading cause of cancer mortality in women worldwide. High-risk human papillomavirus infection is a major cause of cervical cancer. A previous study revealed the role of different oncogenes and tumor suppressors in cervical cancer initiation and progression. However, the complicated genetic network regulating cervical cancer remains largely unknown. The present study reported transcriptome sequencing analysis of three cervical squamous cell cancer tissues and paired normal cervical tissues. Transcriptomic analysis revealed that 2,519 genes were differently expressed between cervical cancer tissues and their corresponding normal tissues. Among these, 236 differentially expressed genes (DEGs) were statistically significant, including many DEGs that were novel in cervical cancer, including gastrulation brain homeobox 2,5-hydroxytryptamine receptor 1D and endothelin 3. These 236 significant DEGs were highly enriched in 28 functional gene ontology categories. The Kyoto Encyclopedia of Genes and Genomes pathway enrichment analysis suggested involvement of these DEGs in multiple pathways. The present study provides a transcriptome landscape of cervical cancer in Chinese patients and an improved understanding of the genetic regulatory network in cervical cancer tumorigenesis.
\end{abstract}

\section{Introduction}

There were 485,000 women newly diagnosed with cervical cancer worldwide in 2013, leading to 236,000 mortalities (1). The incidence and mortality of cervical cancer exhibits an

Correspondence to: Dr Yunbin Ye, Laboratory of ImmunoOncology, Fujian Cancer Hospital and Fujian Medical University Cancer Hospital, 420 Fuma Road, Fuzhou, Fujian 350014, P.R. China E-mail: zjyunbin@sina.com

Key words: transcriptome analysis, cervical squamous cancer, RNA sequencing, differentially expressed genes uneven distribution across the world; almost $85 \%$ of cases are identified in less developed countries. Cervical cancer ranks second among the most diagnosed cancers and is the third leading cause of mortality from cancer in females in less developed countries (2).

It is well known that infection with human papilloma virus is a major cause of cervical cancer (3). Although well-established screening programs and intervention systems have significantly reduced the incidence of cervical cancer in developed countries, patients are still diagnosed with advanced cancer. Limited treatment options for patients with advanced stages of cervical cancer results in a high recurrence rate and a poor prognosis (2). Currently, the combination of cisplatin chemotherapy and radiotherapy is the most common treatment strategy for advanced cervical cancer patients; however, the 5 -year survival rate remains poor $(<50 \%)$. Since the development of pharmacogenomics and personalized medicine, target therapy has dramatically increased the survival rate of cancer patients (4). Treatment of advanced cervical cancers would drastically benefit from the identification of novel therapeutic targets.

With the development of next generation sequencing technologies, RNA sequencing (RNA-Seq) has become a powerful tool for analyzing cancer transcriptomes, detecting such items as alternative splicing, isoform usage, gene fusions and novel transcripts with greater accuracy and higher efficiency $(5,6)$. Initially, RNA-Seq was applied in the expression profile of yeast (7) and human embryonic kidney and B cell lines (8). RNA-Seq has wide application in transcriptome profile analysis in multiple cancers including breast (9) and colon cancers (10). However, the genomic landscape of cervical squamous cell cancer has yet to be elucidated.

To provide an improved understanding of the transcriptome of cervical squamous cell cancer, RNA-Seq of three cervical squamous cell cancer and matched normal tissues was performed. Differentially expressed genes (DEGs) were identified by statistical analysis, and a subset of novel DEGs were validated by reverse transcription-quantitative polymerase chain reaction (RT-qPCR). In addition, Gene Ontology (GO) and Kyoto Encyclopedia of Genes and Genomes (KEGG) pathway enrichment analysis was performed. 


\section{Materials and methods}

Patient samples. For human tissue samples, 27 cervical squamous tumor samples (stage Ib-stage IIb) and matched adjacent normal tissues were obtained from female patients undergoing curative surgery at Fujian Cancer Hospital from April, 2013 to February, 2014 (Fuzhou, China). The median age of the patients was 49 years, (range, 31-59 years). The number of patients in each staging was as follows: Stage Ib (10), stage IIa (12), stage IIb (5). None of the patients received preoperative adjuvant radiotherapy or chemotherapy. All the samples and matched clinical information were collected following written informed consent from the patients.

$R N A$ isolation and $R T-q P C R$. Total RNA from human cervical squamous cancer tissues and corresponding normal tissues was prepared using TRIzol reagent (Invitrogen; Thermo Fisher Scientific, Inc., Waltham, MA, USA) according to the manufacturer's protocol. The RNA quality was determined using an Agilent 2100 Bioanalyzer (Agilent Technologies Inc., Santa Clara, CA, USA). Only RNA extracts with the following criteria were used for RNA-Seq analysis: RNA integrity number, $\geq 7 ; 28 \mathrm{~S} / 18 \mathrm{~S}$ ratio, $>1.8$; OD range, 1.9-2.1. For reverse transcription, cDNA was synthesized using the ReverTra Ace qPCR RT kit (Toyobo Co., Ltd., Osaka, Japan) with $1 \mu \mathrm{g}$ of total RNA. qPCR was performed using the SYBR Select Master Mix for CFX (Invitrogen; Thermo Fisher Scientific, Inc.). The reaction consisted of 1 cycle at $95^{\circ} \mathrm{C}$ for $15 \mathrm{~min}$, followed by 40 cycles of $95^{\circ} \mathrm{C}$ for $15 \mathrm{sec}$, $55^{\circ} \mathrm{C}$ for $30 \mathrm{sec}$ and $70^{\circ} \mathrm{C}$ for $30 \mathrm{sec}$. Primer sequences are presented in Table I. Relative quantification was achieved by normalization to the amount of GAPDH using the $2^{-\Delta \Delta C q}$ method (11). All measurements were repeated 3 times.

Transcriptome deep sequencing. Total RNA extracted from human samples was treated with DNase I. Upon treatment, mRNA was isolated using Oligo d(T)25 Magnetic Beads (New England Biolabs, Inc., Ipswich, MA, USA). RNA preparation was performed as previously described (12). The mRNA was digested into short fragments and cDNA was synthesized using the mRNA fragments as templates. Short fragments were purified and resolved with elution buffer for end reparation and single nucleotide A (adenine) addition. Then the short fragments were connected with adapters and, following agarose gel electrophoresis, the suitable fragments were selected for the PCR amplification as templates. During the quality control steps, an Agilent 2100 Bioanalyzer (Agilent Technologies, Inc., and an ABI StepOnePlus Real-Time PCR system (Applied Biosystems; Thermo Fisher Scientific, Inc.) were used in quantification and qualification of the sample library. The library was sequenced using an Illumina HiSeq ${ }^{\text {TM }} 2000$ (Illumina, Inc., San Diego, CA, USA).

Screening of DEGs. The method for screening of DEGs was developed by Beijing Genomics Institute (BGI, Shenzhen, China) based on a pervious study (13).

$$
p(x)=\frac{e^{-\lambda} \lambda^{x}}{x !}(\lambda \text { is the real trasscripts of the gene) }
$$

The present study defined the number of unambiguous clean tags (which means reads in RNA-Seq) from gene A as $\mathrm{x}$ and, given that every gene's expression occupies only a small part of the library, x yields to the Poisson distribution.

The total clean tag number of the sample 1 is $\mathrm{N} 1$ and the total clean tag number of sample 2 is N2; gene A holds $\mathrm{x}$ tags in sample 1 and y tags in sample 2 . The probability of gene A expressed equally between the two samples can be calculated with:

$$
\begin{gathered}
2 \sum_{i=0}^{i=y} p(i \mid x) \\
\operatorname{or} 2 \times\left(1-\sum_{i=0}^{i=y} p(i \mid x)\right)\left(i f \sum_{i=0}^{k=y} p(i \mid x)>0.5\right) \\
p(y \mid x)=\left(\frac{N_{2}}{N_{1}}\right)^{y} \frac{(x+y) !}{x ! y !\left(1+\frac{N_{2}}{N_{1}}\right)^{(x+y+1)}}
\end{gathered}
$$

A P-value corresponds to the differential gene expression test. Since DEG analysis generates large multiplicity problems in which thousands of hypotheses (as in whether gene $\mathrm{x}$ is differentially expressed between the two groups) are tested simultaneously, a correction for false positive (type I errors) and false negative (type II) errors was performed using the false discovery rate (FDR) method (14). This method assumed that $\mathrm{R}$ differentially expressed genes have been selected in which $\mathrm{S}$ genes really demonstrate differential expression and the other $\mathrm{V}$ genes are false positives. If it is decided that the error ratio ' $\mathrm{Q}=\mathrm{V} / \mathrm{R}$ ' must stay below a cutoff (e.g. 5\%), then the FDR should be preset to a number no larger than 0.05 . FDR $\leq 0.001$ (14) was used and the absolute value of $\log _{2}$ Ratio $\geq 1$ as the threshold to judge the significance of a gene expression difference.

GO analysis of DEGs. All DEGs were mapped to GO terms in the database (http://www.geneontology.org/), calculating gene numbers for every term. Then a hypergeometric was used for the test to find significantly enriched GO terms in the input list of DEGs, based on 'GO:TermFinder' (http://search.cpan. org/dist/GO-TermFinder). Then a strict method was developed to perform this analysis:

$$
\mathrm{P}=1-\sum_{i=0}^{m-1} \frac{\left(\begin{array}{c}
M \\
i
\end{array}\right)\left(\begin{array}{c}
N-M \\
n-i
\end{array}\right)}{\left(\begin{array}{l}
N \\
n
\end{array}\right)}
$$

Where $\mathrm{N}$ is the number of all genes with $\mathrm{GO}$ annotation; $\mathrm{n}$ is the number of DEGs in $\mathrm{N} ; \mathrm{M}$ is the number of all genes that are annotated to certain GO terms; and, $\mathrm{m}$ is the number of DEGs in $\mathrm{M}$. The calculated P-value was adjusted through a Bonferroni Correction (15), and a corrected P-value $\leq 0.05$ was used as a threshold. GO terms fulfilling this condition were defined as significantly enriched GO terms in DEGs.

Pathway enrichment analysis of DEGs. The formula was the same as that in $\mathrm{GO}$ analysis. Here $\mathrm{N}$ is the number of all genes with KEGG annotation, $\mathrm{n}$ is the number of DEGs in $\mathrm{N}$, $\mathrm{M}$ is the number of all genes annotated to specific pathways and $\mathrm{m}$ is the number of DEGs in M.

Statistical analysis. Results from qPCR were analysed using SPSS version 19.0 (SPSS Corp., Armonk, NY, USA). Student's 
Table I. Primers used for reverse transcription quantitative polymerase chain reaction.

\begin{tabular}{lll}
\hline Genes & \multicolumn{1}{c}{ Forward primer } & \multicolumn{1}{c}{ Reverse primer } \\
\hline PRAC & 5'-ATTCTGGTCCCCACCTTTGC-3' & 5'-GGAGGTAGTAAGATGGGCCG-3' \\
EDN3 & 5'-AGACGGTGCCCTATGGACT-3' & 5'GGTCCTTGACTTCAACCTCCTT-3' \\
SOSTDC1 & 5'-AAGTGCAAGAGGTACACCCG-3' & 5'-GGCTCTTTTCCGCTCTCTGT-3' \\
KLK12 & 5'-GTAACCAGCAGCGTTCAACC-3' & 5'-AGGTGGAGTTGCAAATATAGGT-3' \\
KLK13 & 5'-AGCAGGTGAGGGAAGTTGTC-3' & 5'-GGATGGATTTGACCCCCTGG-3' \\
KLK5 & 5'-TTTTCAGAGTCCGTCTCGGC-3' & 5'-GGAACTCTCCCTTTGCCGAA-3' \\
KLK6 & 5'-ATAAGTTGGTGCATGGCGGA-3' & 5'-GTAGATCTCGCTCACCAGTCG-3' \\
OLIG2 & 5'-TCAAGATCAACAGCCGCGAG-3' & 5'-GTAGTCCACATCGCTCTCCA-3' \\
GBX2 & 5'-AGGGCAAGGGAAAGACGAGT-3' & 5'-GCTGCATGACGTTGTCTGTG-3' \\
MUC16 & 5'-TGAGGAGAACATGTGGCCTG-3' & 5'-TTCTGTGCCTCTGAACCCATC-3' \\
CCL1 & 5'-CAGCTCCATCTGCTCCAATGA-3' & 5'-CAGAGCCTGTGATGAGGTGG-3' \\
HTR1D & 5'-CCCTCGGTGTTGCTCATCAT-3' & 5'-AGGGATGATGTTCTGGAGA-3'
\end{tabular}

PRAC; PRAC1 small nuclear protein; EDN3, endothelin 3; SOSTDC1, sclerostin domain containing 1; KLK, kallikrein related peptidase; OLIG2, oligodendrocyte transcription factor 2; GBX2, gastrulation brain homeobox 2; MUC16, mucin 16; CCL1, C-C motif chemokine ligand 1; HTR1D, 5-hydroxytryptamine receptor 1D.

t-test was used to compare the differences in expression between cancer and normal tissues. Cluster analysis was performed using Cluster 3.0 (http://bonsai.hgc.jp/ mdehoon/software/cluster/software.htm); the R version 3.4.0 (R Foundation) with heatmap package (https://stat.ethz.ch/R-manual/ R-devel/library/stats/html/heatmap.html) was applied to the Pearson and Spearman clustering analysis. $\mathrm{P}<0.05$ was considered to indicate a statistically significant result.

\section{Results}

Basic analysis of sequencing data. A basic analysis of sequencing data was performed, whereby the data was compared with the human genome using TopHat version 2.0.9 (https://ccb.jhu.edu/software/tophat/index.shtml); 48.47, 48.79 and 48.77 million clean reads were obtained from cervical squamous cell cancer samples 1 (stage I b), 2 (stage II a) and 3 (stage II b) with genome map rates (proportion of reads mapped to a reference genome) of $85.04,84.74$ and $83.79 \%$, respectively. From the matched normal cervical samples 1-3, 47.31, 48.30 and 47.54 million clean reads were obtained with genome map rates of $86.90,86.33$ and $86.03 \%$ respectively.

Analysis of DEGs. Next, the DEGs between cancer and normal tissue samples were screened. The gene expression level was normalized and measured by reads per $\mathrm{kb}$ of exon per million fragments mapped as described above. In total, 7,936 DEGs were detected in cancer/normal sample 1, 9,077 DEGs in cancer/normal sample 2, and 5,878 DEGs in cancer/normal sample 3 (Fig. 1A). Then, the overlapping DEGs in three pairs of samples were calculated and resulted in 2,519 overlapping DEGs in which 1,450 genes were consistently upregulated in the three cancer samples and 554 genes were consistently downregulated in the three cancer samples (Fig. 1B). However, the rest of the 515 DEGs were not consistently expressed in all three pairs of samples (Fig. 1B).
Cluster analysis revealed that 2,519 DEGs were identical in the three pairs of samples (Fig. 1C). To screen for the statistically significant DEGs, DEGs with fold changes $\geq 3$ were filtered out. With this threshold set, a total of 236 significant DEGs were detected in the three sample pairs, among which 84 DEGs were consistently upregulated and 152 DEGs were consistently downregulated (Table II).

Validation of significant DEGs. To validate the transcriptome analysis data, RT-qPCR was performed on cancer and normal tissue samples to examine the mRNA expression levels for several DEGs identified in the present study. First, established oncogenes and tumor suppressor genes in other types of cancers were examined; gastrulation brain homeobox 2 (GBX2), mucin 16 (MUC16), C-C motif chemokine ligand 1 (CCL1), 5-hydroxytryptamine receptor 1D (HTR1D) and oligodendrocyte transcription factor 2 (OLIG2) are putative oncogene candidates, while PRAC1 small nuclear protein (PRAC), endothelin 3 (EDN3), sclerostin domain containing 1 (SOSTDC1), kallikrein related peptidase (KLK) 12, KLK5, KLK6 and KLK13 are putative tumor suppressors. The mRNA expression levels for these genes were validated in the 27 pairs of cervical squamous cancer samples and matched normal tissue samples. As demonstrated in Fig. 2, expression of GBX2, MUC16, CCL1 and HTR1D was significantly upregulated in cancer samples compared with normal tissues, while expression of PRAC, EDN3, SOSTDC1, KLK12, KLK5, KLK6 and KLK13 was significantly downregulated in cancer samples compared with normal tissues. However, no significance change in expression of OLIG2 was observed in the 27 pairs of samples analysed. OLIG2 is a basic helix-loop-helix transcription factor expressed in the developing central nervous system (CNS) and the postnatal brain (16). OLIG2 is highly expressed in glioblastoma cells, and in glioblastoma initiating cells in particular (16), while expression of OLIG2 is barely detected in other types of cancer. Tissue-specific expression of OLIG2 
A

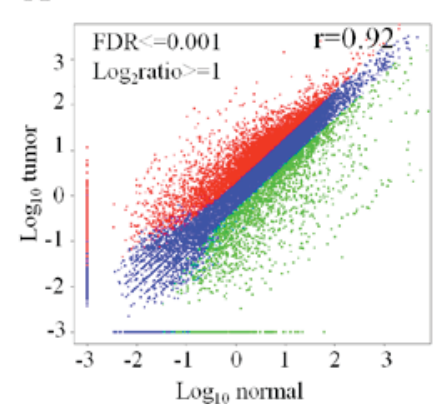

Sample 1

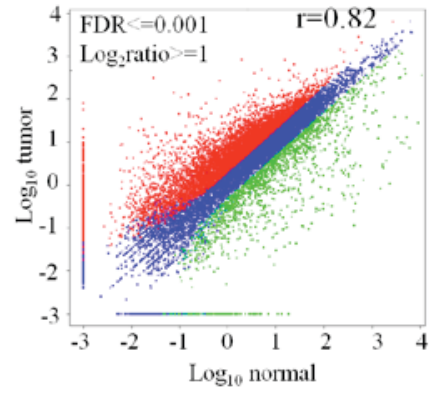

Sample 2

Up-regulated tags

Down-regulated tags

Not differential expressed tag:

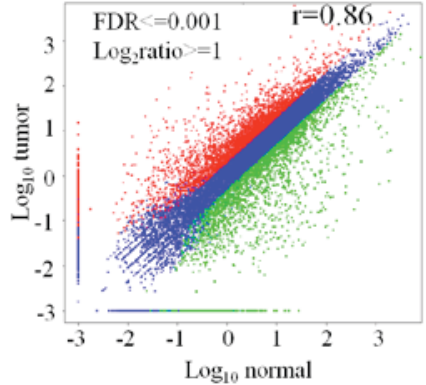

Sample 3
B

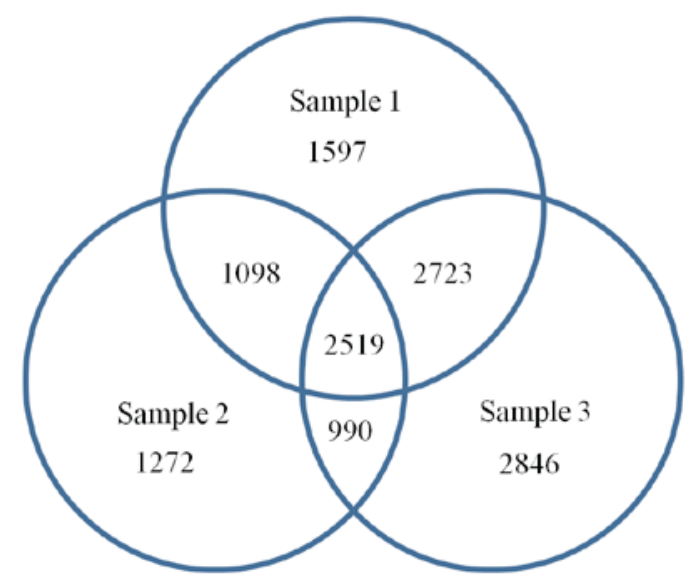

$\mathrm{C}$

Hierarchical cluster of DEGs

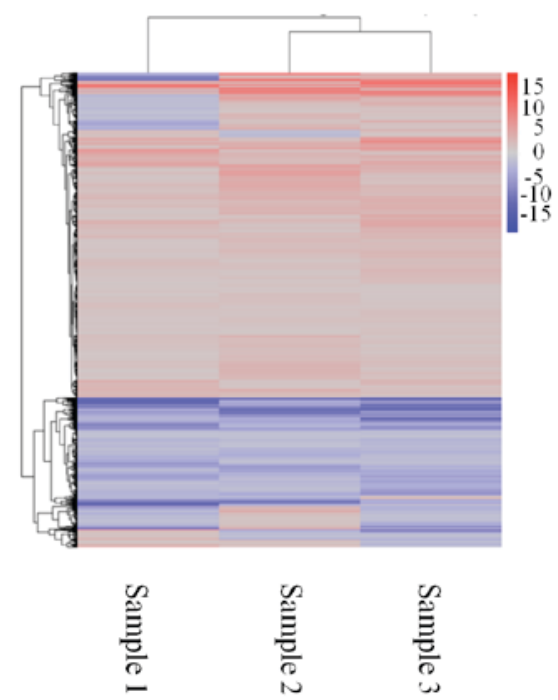

Figure 1. Analysis of differentially expressed genes in cervical squamous cancer tissues and matched normal tissues. (A) Scatter plots of global expression between cancer and normal samples 1-3 were produced by Pearson correlation coefficient analysis. (B) Venn diagram indicating the overlapping DEGs among the three pairs of samples. (C) Hierarchical clustering of DEGs among cervical cancer tissues and matched normal tissues from the three sample pairs. FDR, false discovery rate; DEGs, differentially expressed genes.

in the CNS may explain why no significant change in OLIG2 expression was observed in cervical cancer samples.

Functional enrichment analysis of DEGs. Next, GO analysis of DEGs was performed to obtain a more comprehensive understanding of gene-related biological functions. All DEGs were divided into three major categories based on GO annotations: Biological processes, cellular components and molecular functions. The present study identified that the 236 DEGS were classified into 28 functional categories (Table III); 13 in biological processes, 9 in molecular functions and 6 in cellular components including reproduction, cellular processes and metabolic processes $(\mathrm{P}<0.05$; Fig. 3$)$.

Pathway analysis of DEGs. To understand the functional pathways of significant DEGs, pathway analysis was performed. The 236 significant DEGs (188 with pathway annotation) were involved in numerous key pathways in cancer, including cytokine-cytokine receptor interactions, metabolism of xenobiotics by cytochrome P450 and retinol metabolism (Table IV). A total of 10 significant pathways was detected. The cytokine-cytokine receptor pathway includes numerous chemokines which can link inflammation to cancer. Cytochrome P450 members, which have been linked to carcinogenesis, were also detected. The DEGs involved in these pathways may serve important roles in cervical cancer.

\section{Discussion}

Tumor initiation and progression is a multiple pathway, complicated process, comprising of various dynamic changes in the genome. These genetic alternations contribute to the malignant transformation of cells from normal to cancerous, in addition to tumor progression and metastasis. Malignant cells can acquire favorable genotypes through various biological processes; genetic mutations, epigenetic modifications and non-mutational regulation of gene expression (17). Therefore, genome instability and genetic mutation can be regarded as a 
Table II. List of significant differentially expressed genes.

\begin{tabular}{|c|c|c|c|c|}
\hline Gene symbol & $\begin{array}{c}\log _{2} \\
\text { (cancer vs. normal 1) }\end{array}$ & $\begin{array}{c}\log _{2} \\
\text { (cancer vs. normal 2) }\end{array}$ & $\begin{array}{c}\log _{2} \\
\text { (cancer vs. normal 3) }\end{array}$ & Average \\
\hline GRP & 10.54868 & 9.411035 & 8.89707 & 9.61893 \\
\hline NCRNA00313 & 9.817818 & 9.227841 & 9.280222 & 9.44196 \\
\hline GBX2 & 8.452149 & 7.907159 & 11.89702 & 9.418777 \\
\hline DUSP5P & 9.723775 & 8.01832 & 9.378824 & 9.040307 \\
\hline OSTCL & 8.224955 & 9.28938 & 9.012454 & 8.842263 \\
\hline SLC1A6 & 11.25474 & 6.720402 & 8.382081 & 8.785743 \\
\hline MUC16 (CA125) & 9.088256 & 7.442924 & 9.262444 & 8.597873 \\
\hline C8orf39 & 11.38619 & 10.93562 & 3.461771 & 8.594527 \\
\hline CCL1 & 4.438704 & 9.430026 & 11.37549 & 8.41474 \\
\hline HTR1D & 9.43695 & 7.39446 & 7.546875 & 8.126093 \\
\hline SLFN12L & 5.035945 & 9.239662 & 9.971725 & 8.082443 \\
\hline EN2 & 8.56846 & 5.597178 & 9.531558 & 7.899067 \\
\hline OLIG2 & 6.906961 & 8.928602 & 7.520431 & 7.78533 \\
\hline CAMP & 9.640688 & 9.946121 & 3.303074 & 7.62996 \\
\hline SALL4 & 8.219391 & 10.70523 & 3.458123 & 7.460917 \\
\hline TMED8 & 3.514758 & 12.33337 & 6.105627 & 7.31792 \\
\hline FOXD3 & 3.729651 & 8.969243 & 9.12116 & 7.27335 \\
\hline DNAJC5B & 9.583564 & 8.162562 & 3.478845 & 7.07499 \\
\hline MMP3 & 7.707342 & 9.060809 & 4.216957 & 6.995037 \\
\hline ONECUT2 & 8.016706 & 5.936664 & 6.709699 & 6.88769 \\
\hline SLC24A2 & 7.428435 & 4.883445 & 8.076839 & 6.79624 \\
\hline XIRP1 & 4.323227 & 6.049513 & 9.572409 & 6.648383 \\
\hline TUBA3D & 4.153302 & 7.926962 & 7.772893 & 6.61772 \\
\hline TM7SF4 & 7.775048 & 7.820021 & 4.203538 & 6.599537 \\
\hline FOXD1 & 6.305949 & 8.09621 & 4.807546 & 6.403233 \\
\hline DNAH5 & 7.543471 & 8.300234 & 3.291846 & 6.378517 \\
\hline ZBED6 & 3.223498 & 8.059951 & 7.649948 & 6.311133 \\
\hline INHBA & 5.49018 & 5.640679 & 7.472004 & 6.200953 \\
\hline COL10A1 & 4.945116 & 9.64287 & 3.992286 & 6.193423 \\
\hline CCL18 & 7.794751 & 7.065377 & 3.527205 & 6.12911 \\
\hline HIST1H3G & 3.986192 & 10.74443 & 3.33624 & 6.022287 \\
\hline ESM1 & 5.17567 & 7.531932 & 5.246043 & 5.984547 \\
\hline PLA2G2F & 7.136285 & 7.286441 & 3.465107 & 5.96261 \\
\hline GLDC & 5.663359 & 7.425411 & 4.524814 & 5.871193 \\
\hline CSF2 (GMCSF) & 3.153302 & 10.14843 & 4.162211 & 5.821313 \\
\hline GAS2L3 & 4.311731 & 8.448034 & 4.498861 & 5.752877 \\
\hline FAM172BP & 3.960657 & 8.749995 & 4.269126 & 5.659927 \\
\hline SCN8A & 8.282632 & 4.251928 & 4.299341 & 5.6113 \\
\hline IL24 & 6.704049 & 5.178865 & 4.448351 & 5.443753 \\
\hline AIM2 & 3.001299 & 9.176301 & 3.688599 & 5.288733 \\
\hline KLHDC7B & 3.522536 & 6.009268 & 6.234041 & 5.25528 \\
\hline DSCR6 & 8.119086 & 4.197481 & 3.278907 & 5.19849 \\
\hline CXCL5 & 5.389794 & 5.913205 & 4.16949 & 5.157497 \\
\hline TNNI3 & 4.841802 & 5.316369 & 5.269126 & 5.142433 \\
\hline C1QL1 & 5.261826 & 3.796676 & 6.166828 & 5.07511 \\
\hline DNAH11 & 5.814367 & 4.598159 & 4.538047 & 4.983523 \\
\hline CELSR3 & 4.009208 & 6.425826 & 4.502799 & 4.979277 \\
\hline PTPRR & 3.797158 & 7.448663 & 3.660185 & 4.96867 \\
\hline MGAM & 5.090475 & 4.840449 & 4.606466 & 4.845797 \\
\hline LOC100287559 & 6.917588 & 4.441899 & 3.124736 & 4.828073 \\
\hline ITGB6 & 3.707891 & 7.290941 & 3.246702 & 4.74851 \\
\hline
\end{tabular}


Table II. Continued.

\begin{tabular}{|c|c|c|c|c|}
\hline Gene symbol & $\begin{array}{c}\log _{2} \\
\text { (cancer vs. normal 1) }\end{array}$ & $\begin{array}{c}\log _{2} \\
\text { (cancer vs. normal 2) }\end{array}$ & $\begin{array}{c}\log _{2} \\
\text { (cancer vs. normal 3) }\end{array}$ & Average \\
\hline C1orf152 & 3.551851 & 7.043164 & 3.615894 & 4.73697 \\
\hline VWA3B & 3.908189 & 6.293915 & 3.985333 & 4.729147 \\
\hline EPHB2 & 3.98941 & 6.179193 & 3.841365 & 4.66999 \\
\hline LOC148696 & 3.153302 & 7.298488 & 3.33624 & 4.59601 \\
\hline JPH2 & 3.888979 & 3.351702 & 6.529295 & 4.589993 \\
\hline CDKN2A & 4.664062 & 5.672743 & 3.415469 & 4.58409 \\
\hline TNNT1 & 5.645155 & 4.608629 & 3.449388 & 4.567723 \\
\hline SIM2 & 5.530822 & 4.196787 & 3.783699 & 4.50377 \\
\hline XIST & 3.744393 & 5.117718 & 4.556763 & 4.472957 \\
\hline LOC100775107 & 3.563586 & 5.085756 & 4.595627 & 4.41499 \\
\hline PLOD2 & 4.898069 & 4.881134 & 3.319208 & 4.366137 \\
\hline CNGB 1 & 4.806744 & 4.230395 & 3.852998 & 4.296713 \\
\hline RSAD2 & 3.337828 & 4.916125 & 4.472057 & 4.242003 \\
\hline FLJ43390 & 5.751652 & 3.737355 & 3.046734 & 4.17858 \\
\hline EGFR & 5.416012 & 3.283692 & 3.41214 & 4.03728 \\
\hline LOC 100190986 & 3.642481 & 4.901331 & 3.562375 & 4.035397 \\
\hline FAM63B & 3.302318 & 4.095168 & 4.669664 & 4.022383 \\
\hline UBXN7 & 3.777425 & 4.331877 & 3.822488 & 3.977263 \\
\hline LOC283299 & 3.47523 & 3.682908 & 4.577248 & 3.911797 \\
\hline ARHGAP11B & 4.835126 & 3.582381 & 3.198737 & 3.87208 \\
\hline BCAT1 & 4.92309 & 3.413721 & 3.162697 & 3.83317 \\
\hline IGF2BP2 & 3.48637 & 4.974384 & 3.016948 & 3.8259 \\
\hline IFIT3 & 3.33571 & 3.670937 & 4.105221 & 3.703957 \\
\hline IFIT2 & 4.388168 & 3.335629 & 3.319752 & 3.681183 \\
\hline GALNT4 & 4.730731 & 3.012215 & 3.124736 & 3.62256 \\
\hline ATP13A3 & 3.28667 & 4.347695 & 3.224075 & 3.61948 \\
\hline TRIO & 3.506362 & 3.683108 & 3.63553 & 3.608333 \\
\hline KIAA0895 & 3.323227 & 3.2336 & 4.08545 & 3.547427 \\
\hline PTPLB & 3.033159 & 4.04489 & 3.517358 & 3.531803 \\
\hline WDR31 & 3.503799 & 3.477879 & 3.509077 & 3.496917 \\
\hline ASPHD1 & 3.438704 & 3.304396 & 3.282801 & 3.341967 \\
\hline FLJ34208 & 3.612733 & 3.230395 & 3.124736 & 3.322622 \\
\hline IL1B & 3.113062 & 3.39834 & 3.179301 & 3.230234 \\
\hline PRAC & -13.4151 & -13.6146 & -12.5618 & -13.1972 \\
\hline EDN3 & -7.12592 & -15.8836 & -11.4601 & -11.4899 \\
\hline FABP12 & -11.8695 & -10.8052 & -10.1364 & -10.937 \\
\hline LOC644759 & -12.1577 & -10.3962 & -10.1672 & -10.907 \\
\hline KLK12 & -9.45888 & -9.66283 & -12.3341 & -10.4853 \\
\hline SOSTDC1 & -5.56369 & -13.3193 & -11.2345 & -10.0392 \\
\hline PROK1 & -6.99645 & -8.77908 & -13.103 & -9.62617 \\
\hline NCRNA00160 & -10.4406 & -9.28356 & -8.65661 & -9.46027 \\
\hline HS3ST6 & -4.58141 & -14.2018 & -9.36341 & -9.3822 \\
\hline LOC642366 & -9.06419 & -9.70611 & -8.97064 & -9.24697 \\
\hline KLK5 & -6.72497 & -6.72175 & -14.0832 & -9.17663 \\
\hline LGI3 & -9.66722 & -12.6499 & -4.90455 & -9.07387 \\
\hline C18orf26 & -11.4915 & -8.05915 & -7.5957 & -9.0488 \\
\hline KLK6 & -10.1991 & -6.49943 & -10.1097 & -8.93607 \\
\hline FTLP10 & -9.17158 & -9.63008 & -7.80673 & -8.86947 \\
\hline KRT13 & -7.04312 & -9.72684 & -9.80361 & -8.85787 \\
\hline SDR9C7 & -8.99263 & -5.71915 & -11.7824 & -8.8314 \\
\hline
\end{tabular}


Table II. Continued.

\begin{tabular}{|c|c|c|c|c|}
\hline Gene symbol & $\begin{array}{c}\log _{2} \\
\text { (cancer vs. normal 1) }\end{array}$ & $\begin{array}{c}\log _{2} \\
\text { (cancer vs. normal 2) }\end{array}$ & $\begin{array}{c}\log _{2} \\
\text { (cancer vs. normal 3) }\end{array}$ & Average \\
\hline KLK13 & -7.35941 & -9.8484 & -8.89013 & -8.6993 \\
\hline CYP4F22 & -7.35608 & -9.07243 & -9.50559 & -8.6447 \\
\hline CWH43 & -8.37808 & -4.96987 & -12.2752 & -8.54107 \\
\hline UPK1A & -8.75809 & -11.6327 & -5.03608 & -8.47563 \\
\hline SPRR2C & -9.5305 & -6.16292 & -9.61626 & -8.43657 \\
\hline ISL1 & -6.24758 & -11.9118 & -6.85564 & -8.33833 \\
\hline CRNN & -9.03239 & -10.5285 & -5.43238 & -8.3311 \\
\hline TMPRSS11BNL & -12.7646 & -7.64689 & -4.48074 & -8.29743 \\
\hline SPRR3 & -9.08119 & -9.16385 & -6.62833 & -8.29113 \\
\hline MUC21 & -7.66328 & -9.01786 & -7.95629 & -8.21247 \\
\hline SFTA2 & -6.72934 & -6.03851 & -11.348 & -8.0386 \\
\hline KRTDAP & -10.8126 & -9.41425 & -3.83469 & -8.0205 \\
\hline HOXB13-AS1 & -9.26181 & -10.8682 & -3.93055 & -8.0202 \\
\hline SPINK5 & -7.0192 & -8.64938 & -8.34653 & -8.00503 \\
\hline ARSF & -12.2472 & -3.60249 & -8.14556 & -7.99843 \\
\hline KRT4 & -6.28401 & -9.91166 & -7.48655 & -7.89407 \\
\hline MAL & -9.13047 & -9.3469 & -5.19779 & -7.89173 \\
\hline SPINK7 & -9.40574 & -9.496 & -4.58648 & -7.8294 \\
\hline KLK11 & -5.43434 & -9.29366 & -8.63959 & -7.7892 \\
\hline LOC 144817 & -3.11972 & -10.3462 & -9.80503 & -7.757 \\
\hline SPRR1B & -8.95135 & -5.98911 & -7.93974 & -7.62673 \\
\hline SBSN & -8.96791 & -9.33194 & -4.57612 & -7.62533 \\
\hline CLCA4 & -5.66679 & -5.92101 & -11.1121 & -7.56663 \\
\hline TMPRSS11A & -5.85846 & -5.25567 & -11.315 & -7.4764 \\
\hline TMPRSS11B & -8.1584 & -9.30062 & -4.81077 & -7.42327 \\
\hline PRSS3 & -8.39642 & -8.84527 & -4.99691 & -7.41287 \\
\hline PBMUCL1 & -8.68117 & -6.30909 & -7.22153 & -7.40393 \\
\hline FADS6 & -7.76112 & -5.07643 & -9.35563 & -7.39773 \\
\hline KLK9 & -8.21271 & -3.01753 & -10.9561 & -7.39543 \\
\hline LRRTM4 & -6.356 & -7.06931 & -8.7054 & -7.3769 \\
\hline ALOX12B & -9.21357 & -8.75139 & -4.12319 & -7.36273 \\
\hline RHCG & -8.60154 & -4.29579 & -8.9801 & -7.29247 \\
\hline RDH12 & -8.76324 & -7.91437 & -5.01249 & -7.23003 \\
\hline TMPRSS11D & -5.68278 & -5.64144 & -10.3586 & -7.2276 \\
\hline KLK10 & -4.85195 & -7.938 & -8.84348 & -7.21113 \\
\hline DUOXA2 & -6.6414 & -7.13884 & -7.7198 & -7.16667 \\
\hline KRT16P1 & -8.39166 & -6.41271 & -6.65457 & -7.15297 \\
\hline WFDC5 & -5.94473 & -3.59185 & -11.6523 & -7.06297 \\
\hline TMPRSS11F & -5.56643 & -4.27303 & -11.237 & -7.0255 \\
\hline KRT78 & -8.71162 & -9.09812 & -3.24017 & -7.01663 \\
\hline CYP2C18 & -5.67597 & -9.10234 & -6.21065 & -6.99633 \\
\hline ENDOU & -8.373 & -6.00788 & -6.5908 & -6.99057 \\
\hline TMPRSS11E & -7.36594 & -4.83162 & -8.75233 & -6.9833 \\
\hline KLK8 & -5.28042 & -7.14018 & -8.49714 & -6.97257 \\
\hline SLURP1 & -7.64971 & -9.05043 & -4.11415 & -6.9381 \\
\hline HOXB 13 & -11.8912 & -3.76729 & -5.14309 & -6.93387 \\
\hline NR0B1 & -3.16863 & -8.78707 & -8.8382 & -6.9313 \\
\hline DSG1 & -7.27882 & -9.56852 & -3.70815 & -6.85183 \\
\hline OLFM4 & -4.41224 & -4.86631 & -11.2427 & -6.84043 \\
\hline ASPG & -9.35845 & -5.80053 & -5.34926 & -6.83607 \\
\hline SPRR2F & -11.0558 & -5.91653 & -3.44512 & -6.8058 \\
\hline
\end{tabular}


Table II. Continued.

\begin{tabular}{|c|c|c|c|c|}
\hline Gene symbol & $\begin{array}{c}\log _{2} \\
\text { (cancer vs. normal 1) }\end{array}$ & $\begin{array}{c}\log _{2} \\
\text { (cancer vs. normal 2) }\end{array}$ & $\begin{array}{c}\log _{2} \\
\text { (cancer vs. normal 3) }\end{array}$ & Average \\
\hline HCG22 & -6.72322 & -9.14481 & -4.41865 & -6.76223 \\
\hline KRT16P3 & -6.29516 & -6.68591 & -7.20534 & -6.7288 \\
\hline CPA6 & -4.97598 & -3.57612 & -11.5417 & -6.69793 \\
\hline KRT6C & -7.20141 & -6.9751 & -5.85838 & -6.6783 \\
\hline SCEL & -7.31534 & -4.10998 & -8.50819 & -6.6445 \\
\hline CRHR1 & -4.20425 & -8.6737 & -6.95224 & -6.61007 \\
\hline KLK7 & -4.16482 & -7.54068 & -8.07971 & -6.59507 \\
\hline FABP4 & -3.60159 & -10.8444 & -5.24657 & -6.5642 \\
\hline C18orf34 & -3.43166 & -8.03628 & -7.85036 & -6.43943 \\
\hline IVL & -9.68789 & -3.53827 & -6.07053 & -6.43223 \\
\hline SPRR2A & -8.33607 & -6.76353 & -4.16411 & -6.42123 \\
\hline GJB6 & -5.78401 & -4.68417 & -8.7729 & -6.4137 \\
\hline SPRR1A & -8.69142 & -5.35853 & -5.18329 & -6.41107 \\
\hline CEACAM7 & -3.8467 & -6.03077 & -9.32939 & -6.4023 \\
\hline PPP1R3C & -6.54484 & -9.23918 & -3.33717 & -6.37373 \\
\hline C2orf54 & -8.04377 & -5.14492 & -5.76023 & -6.3163 \\
\hline CCL14 & -3.97598 & -11.2998 & -3.62144 & -6.29907 \\
\hline PRSS27 & -8.34943 & -4.60582 & -5.83476 & -6.26333 \\
\hline FUT6 & -4.6948 & -6.47518 & -7.48074 & -6.2169 \\
\hline LRP1B & -4.69219 & -8.09952 & -5.84579 & -6.2125 \\
\hline KCNK10 & -4.25851 & -6.50135 & -7.80251 & -6.18747 \\
\hline WISP2 & -5.54635 & -6.65683 & -6.06 & -6.08773 \\
\hline SPRR2D & -8.64649 & -6.06525 & -3.46049 & -6.0574 \\
\hline CYP2B7P1 & -4.29557 & -6.36724 & -7.48514 & -6.0493 \\
\hline CLDN8 & -3.1579 & -5.95613 & -8.77604 & -5.96337 \\
\hline GDF6 & -4.05615 & -7.32225 & -6.49891 & -5.9591 \\
\hline TMEM45B & -5.61223 & -5.92896 & -6.3174 & -5.95287 \\
\hline NEFL & -4.20425 & -3.92442 & -9.62952 & -5.9194 \\
\hline DAPL1 & -5.71856 & -4.88481 & -7.14556 & -5.9163 \\
\hline FAM3D & -5.63608 & -4.61094 & -7.45818 & -5.90173 \\
\hline C10orf99 & -5.66475 & -3.59184 & -8.361 & -5.87253 \\
\hline ATP13A4 & -4.13757 & -6.36183 & -7.11188 & -5.87043 \\
\hline PSCA & -8.80031 & -5.31464 & -3.37421 & -5.82973 \\
\hline GSTM5 & -4.86629 & -7.56056 & -4.82363 & -5.75017 \\
\hline CRYM & -4.82557 & -7.89036 & -4.42697 & -5.7143 \\
\hline ALOX12 & -6.72993 & -7.05622 & -3.27911 & -5.68843 \\
\hline LYPD2 & -5.20351 & -6.50013 & -5.28866 & -5.6641 \\
\hline DEGS2 & -3.47443 & -6.45172 & -6.87474 & -5.6003 \\
\hline TP53AIP1 & -4.83451 & -6.02192 & -5.90829 & -5.58823 \\
\hline APOD & -4.46756 & -8.75006 & -3.50099 & -5.57287 \\
\hline PLA2G4F & -5.6344 & -3.88007 & -7.0168 & -5.51043 \\
\hline TMEM132C & -3.09463 & -10.0647 & -3.37112 & -5.51017 \\
\hline GJB2 & -6.73282 & -3.11385 & -6.60454 & -5.48373 \\
\hline PTGDS & -5.8487 & -6.56155 & -4.02011 & -5.4768 \\
\hline NCCRP1 & -6.9491 & -3.38815 & -6.05232 & -5.4632 \\
\hline KRT16P2 & -4.82398 & -5.03598 & -6.48074 & -5.4469 \\
\hline DUOX2 & -3.89572 & -4.21314 & -8.14256 & -5.41713 \\
\hline LOC283392 & -3.65906 & -4.6075 & -7.9533 & -5.40663 \\
\hline C21orf15 & -4.96246 & -7.22455 & -4.03008 & -5.4057 \\
\hline HOPX & -4.52883 & -6.72314 & -4.68297 & -5.31163 \\
\hline C7 & -4.26858 & -5.33946 & -6.24421 & -5.28407 \\
\hline
\end{tabular}


Table II. Continued.

\begin{tabular}{|c|c|c|c|c|}
\hline Gene symbol & $\begin{array}{c}\log _{2} \\
\text { (cancer vs. normal 1) }\end{array}$ & $\begin{array}{c}\log _{2} \\
\text { (cancer vs. normal 2) }\end{array}$ & $\begin{array}{c}\log _{2} \\
\text { (cancer vs. normal 3) }\end{array}$ & Average \\
\hline FXYD1 & -4.38736 & -8.16219 & -3.10047 & -5.21667 \\
\hline VSIG10L & -5.64524 & -6.67538 & -3.28431 & -5.20163 \\
\hline CXCL14 & -4.06317 & -4.69872 & -6.79264 & -5.18483 \\
\hline C1orf177 & -7.12468 & -4.89746 & -3.40118 & -5.1411 \\
\hline PGLYRP3 & -4.2413 & -5.30136 & -5.87213 & -5.13827 \\
\hline NDRG4 & -3.11573 & -6.16805 & -6.10851 & -5.13077 \\
\hline SH3GL2 & -3.43166 & -7.88472 & -4.03008 & -5.1155 \\
\hline D4S234E & -6.32648 & -5.24772 & -3.58727 & -5.05383 \\
\hline HSPB6 & -4.66679 & -6.44156 & -3.92896 & -5.01243 \\
\hline KRT32 & -4.88012 & -4.74755 & -5.40859 & -5.0121 \\
\hline C15orf59 & -3.52682 & -5.00472 & -6.41321 & -4.98157 \\
\hline ECM1 & -5.89039 & -4.95876 & -3.74402 & -4.8644 \\
\hline PKNOX2 & -3.80089 & -7.16728 & -3.44512 & -4.80443 \\
\hline SLC22A3 & -5.01115 & -5.30293 & -4.01922 & -4.77777 \\
\hline SCN2B & -3.37919 & -6.85042 & -3.98117 & -4.73693 \\
\hline BNIPL & -3.30443 & -4.13255 & -6.42697 & -4.62133 \\
\hline S100A12 & -5.68204 & -3.48731 & -4.64434 & -4.60457 \\
\hline TPRG1 & -5.48765 & -5.09685 & -3.17304 & -4.58583 \\
\hline F10 & -4.46875 & -5.39257 & -3.72098 & -4.52743 \\
\hline KRT14 & -5.32 & -3.2456 & -5.00968 & -4.5251 \\
\hline PGLYRP4 & -5.26736 & -3.75249 & -4.48259 & -4.5008 \\
\hline CYP2F1 & -5.58817 & -3.60249 & -4.21065 & -4.4671 \\
\hline SCARA5 & -3.8261 & -6.19246 & -3.34042 & -4.453 \\
\hline DNASE1L3 & -4.77494 & -3.71102 & -4.65301 & -4.37967 \\
\hline CD300LG & -5.09807 & -3.14682 & -4.72805 & -4.3243 \\
\hline FABP5 & -3.59716 & -3.02627 & -6.02887 & -4.21743 \\
\hline CORIN & -3.67113 & -5.92442 & -3.04919 & -4.2149 \\
\hline TGM5 & -4.13436 & -3.93665 & -4.13218 & -4.06773 \\
\hline TMPRSS2 & -3.3997 & -3.8615 & -4.73918 & -4.00013 \\
\hline SCN4B & -3.0682 & -4.22699 & -4.44512 & -3.91343 \\
\hline ANXA9 & -3.47853 & -3.71392 & -4.52455 & -3.90567 \\
\hline NEFM & -4.29673 & -3.47696 & -3.66751 & -3.81373 \\
\hline CD1E & -3.4358 & -3.40555 & -3.64926 & -3.49687 \\
\hline
\end{tabular}

hallmark of cancer. With the development of molecular biology and genetics, biologists have gained an improved view of the landscape of the cancer genome by utilizing novel methods including high-throughput genome sequencing. The present study provided a comprehensive transcriptome analysis of cervical squamous cancer tissue with matched normal tissues by RNA-Seq. First, a basic analysis of the sequencing data was performed to detect levels of DEGs. Then, advanced analysis was conducted to give insights into the biological functions and pathways that involved the DEGs. Thus, the present study may aid in discovering novel diagnostic and therapeutic targets for cervical cancer.

The present RNA-Seq analysis acquired $\sim 290$ million reads, a number which is adequate for transcriptome sequencing. Furthermore, the genome map rate of sequencing reads was $\sim 85 \%$, indicating that the sequencing process met the criteria of the initial quality control for RNA-Seq techniques (18). These data suggested that the quality of the experimental method and data processing were sufficient.

The significant DEGs were further analysed to confirm whether the sequencing results were consistent with previous research. Matrix metalloproteinase (MMP)3 has been reported to be upregulated in cervical cancer by microarray analysis and by immunohistochemistry $(19,20)$. MMP3 has also been reported to exhibit higher expression and enzyme activity in breast cancer cells that metastatic to the brain and during epithelial-to-mesenchymal transition (EMT) (21). The data from the present study revealed that MMP3 (Table II) was one of the overexpressed DEGs identified in three different cervical cancer samples compared with normal tissues, which is supported by previous studies $(19,20)$. SIX homeobox 1 (SIX1) protein is a transcription factor regulating cell proliferation, 
Table III. Detailed list of significant DEGs by functional categories from GO analysis.

Functional category

Binding (GO:0005488)

Catalytic activity (GO:0003824)

Enzyme regulator activity (GO:0030234)

Nucleic acid binding transcription factor activity (GO:0001071)

Receptor activity (GO:0004872)

Structural molecule activity (GO:0005198)

Transporter activity (GO:0005215) Apoptotic process (GO:0006915)

Biological adhesion (GO:0022610) Biological regulation (GO:0065007)

Cellular component organization or biogenesis (GO:0071840) Cellular process (GO:0009987)

Developmental process (GO:0032502)

Immune system process (GO:0002376)

Localization (GO:0051179)

Locomotion (GO:0040011) metabolic process (GO:0008152)
Significant DEGs

EDN3, CXCL5, GBX2, LRRTM4, TMPRSS11D, WFDC5, NR0B1, VWA3B, CELSR3, INHBA, ONECUT2, OLIG2, PROK1, TMPRSS11B, KLK10, KLK7, PTGDS, F10, IL1 $\beta$, CCL1, ISL1, SPINK5, GDF6, FOXD3, CRNN, KLK11, TMPRSS11A, KLK9, TMPRSS11F, TMPRSS11E, HOXB13, CCL14, S100A12, IGF2BP2, IL24, CCL18, KLK12, KLK5, BNIPL, TMPRSS2, TNNI3, ESM1, FOXD1, CAMP, SPINK7, PRSS27, WISP2, PGLYRP3, CNGB1, EN2, UPK1A, KLK8, DSG1, PPP1R3C, DAPL1, PGLYRP4, CD1E, TRIO, SIM2, GAS2L3, SALL4

TMPRSS11D, WFDC5, VWA3B, OLIG2, TMPRSS11B, KLK10, KLK7, PTGDS, F10, KLK11, TMPRSS11F, TMPRSS11E, IGF2BP2, KLK12, KLK5, TMPRSS2, CAMP, SPINK7, PRSS27, KLK8, PPP1R3C, DAPL1, TRIO

TMPRSS11D, WFDC5, VWA3B, OLIG2, TMPRSS11B, KLK10, KLK7, PTGDS, F10, KLK11, TMPRSS11F, TMPRSS11E, IGF2BP2, KLK12, KLK5, TMPRSS2, CAMP, SPINK7, PRSS27, KLK8, PPP1R3C, DAPL1, TRIO

GBX2, NR0B1, ONECUT2, OLIG2, ISL1, FOXD3, HOXB13, FOXD1, EN2, SIM2, SALL4

LRRTM4, TMPRSS11D, NR0B1, CELSR3, TMPRSS11B, KLK10, KLK7, F10, KLK11, TMPRSS11A KLK9, TMPRSS11F, TMPRSS11E, KLK12, KLK5, TMPRSS2, PRSS27, UPK1A, KLK8

TNNI3, GAS2L3

\section{CNGB1}

TMPRSS11D, INHBA, TMPRSS11B, GDF6, KLK11, TMPRSS11A, KLK9 TMPRSS11F, TMPRSS11E, IGF2BP2, PGL, YRP3, DAPL1, PGLYRP4 LRRTM4, CELSR3, WISP2, UPK1A

EDN3, CXCL5, GBX2, LRRTM4, MPRSS11D, WFDC5, NR0B1, VWA3B INHBA, ONECUT2, OLIG2, TMPRSS11B, KLK10, KLK7, F10, IL1B, ISL1, GDF6, FOXD3, KLK11, TMPRSS11A, KLK9, TMPRSS11F, TMPRSS11E, HOXB13, IL24, KLK12, KLK5, TMPRSS2, FOXD1, CAMP SPINK7, PRSS27, WISP2, CNGB1, EN2, KLK8, PPP1R3C, DAPL1, TRIO, SIM2, SALL4

CELSR3

EDN3, CXCL5, LRRTM4, CELSR3, INHBA, ONECUT2, PTGDS, IL1B CCL1, GDF6, FOXD3, CRNN, CCL14, S100A12, IGF2BP2, IL24, CCL18, BNIPL, FOXD1, WISP2, CNGB1, UPK1A, DSG1, DAPL1, TRIO, GAS2L3

GBX2, LRRTM4, TMPRSS11D, NR0B1, CELSR3, INHBA, ONECUT2, OLIG2, PROK1, TMPRSS11B, ISL1, GDF6, KLK11, TMPRSS11A, KLK9, TMPRSS11F, TMPRSS11E, HOXB13, IGF2BP2, BNIPL, TMPRSS2, TNNI3, WISP2, PGL, YRP3, EN2, DAPL1, PGLYRP4, SIM2, GAS2L3

CXCL5, TMPRSS11D, TMPRSS11B, KLK10, KLK7, PTGDS, F10, KLK11, TMPRSS11A, KLK9, TMPRSS11F, TMPRSS11E, IGF2BP2, KLK12, KLK5, TMPRSS2, PRSS27, CNGB1, KLK8

CXCL5, TMPRSS11D, TMPRSS11B, KLK10, KLK7, PTGDS, F10, KLK11, TMPRSS11A, KLK9, TMPRSS11F, TMPRSS11E, IGF2BP2, KLK12, KLK5, TMPRSS2, PRSS27, CNGB1, KLK8

CXCL5

GBX2, TMPRSS11D, WFDC5, NR0B1, VWA3B INHBA, ONECUT2, OLIG2, TMPRSS11B, KLK10, KLK7, PTGDS, F10, ISL1, GDF6, FOXD3 CRNN, KLK11, TMPRSS11A, KLK9, TMPRSS11F, TMPRSS11E, HOXB13, S100A12, IGF2BP2, KLK12, KLK5, TMPRSS2, FOXD1, CAMP, SPINK7 PRSS27, PGLYRP3, EN2, KLK8, PPP1R3C, DAPL1 PGLYRP4, TRIO, SIM2. SALL4 
Table III. Continued.

Functional category

Significant DEGs

Multicellular organismal process

(GO:0032501)

Reproduction (GO:0000003)

Response to stimulus (GO:0050896)

Cell part (GO:0044464)

Extracellular matrix (GO:0031012)

Extracellular region (GO:0005576)

Macromolecular complex (GO:0032991)

Membrane (GO:0016020)

Organelle (GO:0043226)
TMPRSS11D, ONECUT2, PROK1, TMPRSS11B, KLK11, TMPRSS11A, KLK9, TMPRSS11F, TMPRSS11E, HOXB13, IGF2BP2, TMPRSS2, TNNI3, UPK1A, TRIO

F10, HOXB13, TMPRSS2, PRSS27, UPK1A

CXCL5, TMPRSS11D, INHBA, TMPRSS11B, KLK10, KLK7, F10, IL1 $\beta$, CCL1, GDF6, KLK11, TMPRSS11A, KLK9, TMPRSS11F, TMPRSS11E, CCL14, S100A12, IL24, CCL18, KLK12, KLK5, TMPRSS2, CAMP, PRSS27, WISP2, CNGB1, UPK1A, KLK8, TRIO

PGLYRP4, TRIO, SIM2, SALL4

LRRTM4, WISP2

CXCL5, LRRTM4, TMPRSS11D, INHBA, TMPRSS11B, KLK10, KLK7, F10, IL1 $\beta$, GDF6, KLK11, TMPRSS11A, KLK9, TMPRSS11F, TMPRSS11E, IL24, KLK12, KLK5, TMPRSS2, PRSS27, WISP2, KLK8

IGF2BP2

CNGB1, DSG1, CD1E

ONECUT2, FOXD3, TNNI3, FOXD1

DEGs, differentially expressed genes; GO, gene ontology.
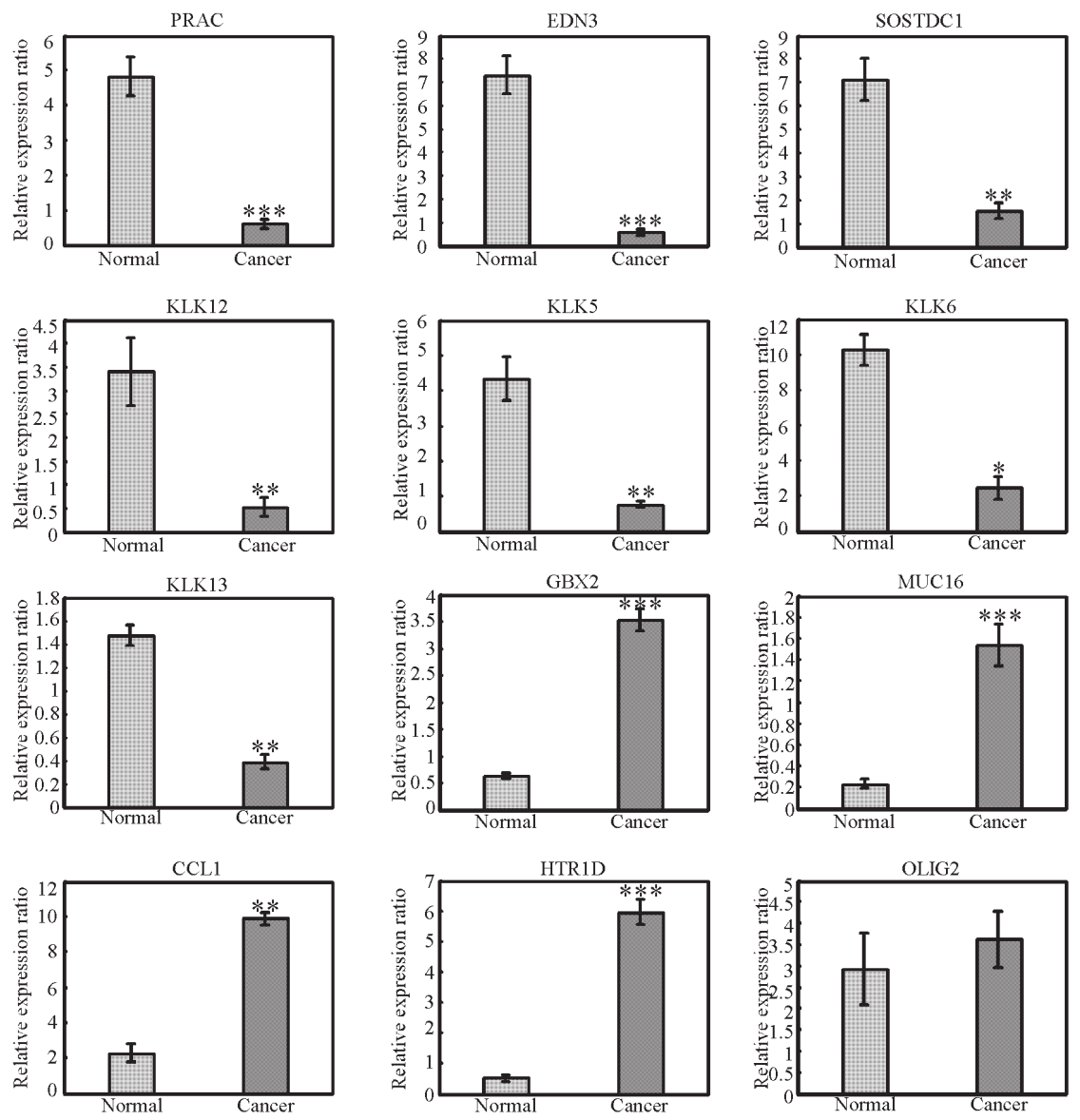

Figure 2. Validation of differentially expressed genes. The mRNA expression levels of PRAC, EDN3, SOSTDC1, KLK12, KLK13, KLK5, KLK6, OLIG2, GBX2, MUC16, CCL1 and HTR1D were examined by reverse transcription-quantitative polymerase chain reaction in the 27 pairs of cancer and matched normal samples. ${ }^{*} \mathrm{P}<0.05,{ }^{* *} \mathrm{P}<0.01$ and ${ }^{* * * *} \mathrm{P}<0.001$ vs. normal. PRAC, PRAC1 small nuclear protein; EDN3, endothelin 3; SOSTDC1, sclerostin domain containing 1; KLK, kallikrein related peptidase; GBX2, gastrulation brain homeobox 2; MUC16, mucin 16; CCL1, C-C motif chemokine ligand 1; HTR1D, 5-hydroxytryptamine receptor 1D; OLIG2, oligodendrocyte transcription factor 2. 
Table IV. KEGG pathway analysis of significant DEGs.

\begin{tabular}{|c|c|c|c|c|}
\hline $\begin{array}{l}\text { KEGG } \\
\text { pathway }\end{array}$ & $\begin{array}{l}\text { Number of } \\
\text { DEGs involved } \\
\text { in pathway }\end{array}$ & $\begin{array}{c}\% \text { of total } \\
\text { DEGs involved } \\
\text { in pathway }\end{array}$ & P-value & DEGs \\
\hline $\begin{array}{l}\text { Arachidonic acid } \\
\text { metabolism }\end{array}$ & 8 & 4.26 & $1.8083 \times 10^{-5}$ & $\begin{array}{l}\text { ALOX12, TMEM242, CYP2B7P, CYP2C18, } \\
\text { CYP4F22, PLA2G2F, PLA2G4F, PTGDS }\end{array}$ \\
\hline $\begin{array}{l}\text { Cytokine-cytokine } \\
\text { receptor interaction }\end{array}$ & 11 & 5.85 & 0.0007 & $\begin{array}{l}\text { CCL1, CCL14, CCL18, CSF2, CXCL14, } \\
\text { CXCL5, EGFR, GDF6, IL-1 } \beta \text {, IL24, INHBA }\end{array}$ \\
\hline $\begin{array}{l}\text { Staphylococcus } \\
\text { aureus infection }\end{array}$ & 7 & 3.72 & 0.0008 & $\begin{array}{l}\text { DSG1, KRT13, KRT14, KRT16P1, KRT16P2, } \\
\text { KRT16P3, KRT32, }\end{array}$ \\
\hline $\begin{array}{l}\text { Pathogenic Escherichia } \\
\text { coli infection }\end{array}$ & 9 & 4.79 & 0.0009 & $\begin{array}{l}\text { CCDC178, CLDN8, KRT13, KRT14, KRT16P1, } \\
\text { KRT16P2, KRT16P3, KRT32, TUBA3D }\end{array}$ \\
\hline Rheumatoid arthritis & 5 & 2.66 & 0.0085 & CCL18, CSF2, CXCL5, IL-1 $\beta$, MMP3 \\
\hline Linoleic acid metabolism & 3 & 1.6 & 0.0137 & CYP2C18, PLA2G2F, PLA2G4F \\
\hline $\begin{array}{l}\text { Metabolism of xenobiotics } \\
\text { by cytochrome P450 }\end{array}$ & 4 & 2.13 & 0.0139 & CYP2B7P, CYP2C18, CYP2F1, GSTM5 \\
\hline Retinol metabolism & 4 & 2.13 & 0.0139 & CYP2B7P, CYP2C18, RDH12, SDR9C7 \\
\hline PPAR signaling pathway & 5 & 2.66 & 0.0153 & C1QL1, COL10A1, FABP12, FABP4, FABP5 \\
\hline $\begin{array}{l}\text { Cytosolic DNA-sensing } \\
\text { pathway }\end{array}$ & 3 & 1.6 & 0.0470 & AIM2, HCG22, IL-1 $\beta$ \\
\hline
\end{tabular}

KEGG, Kyoto Encyclopedia of genes and genomes; DEGs, differentially expressed genes.

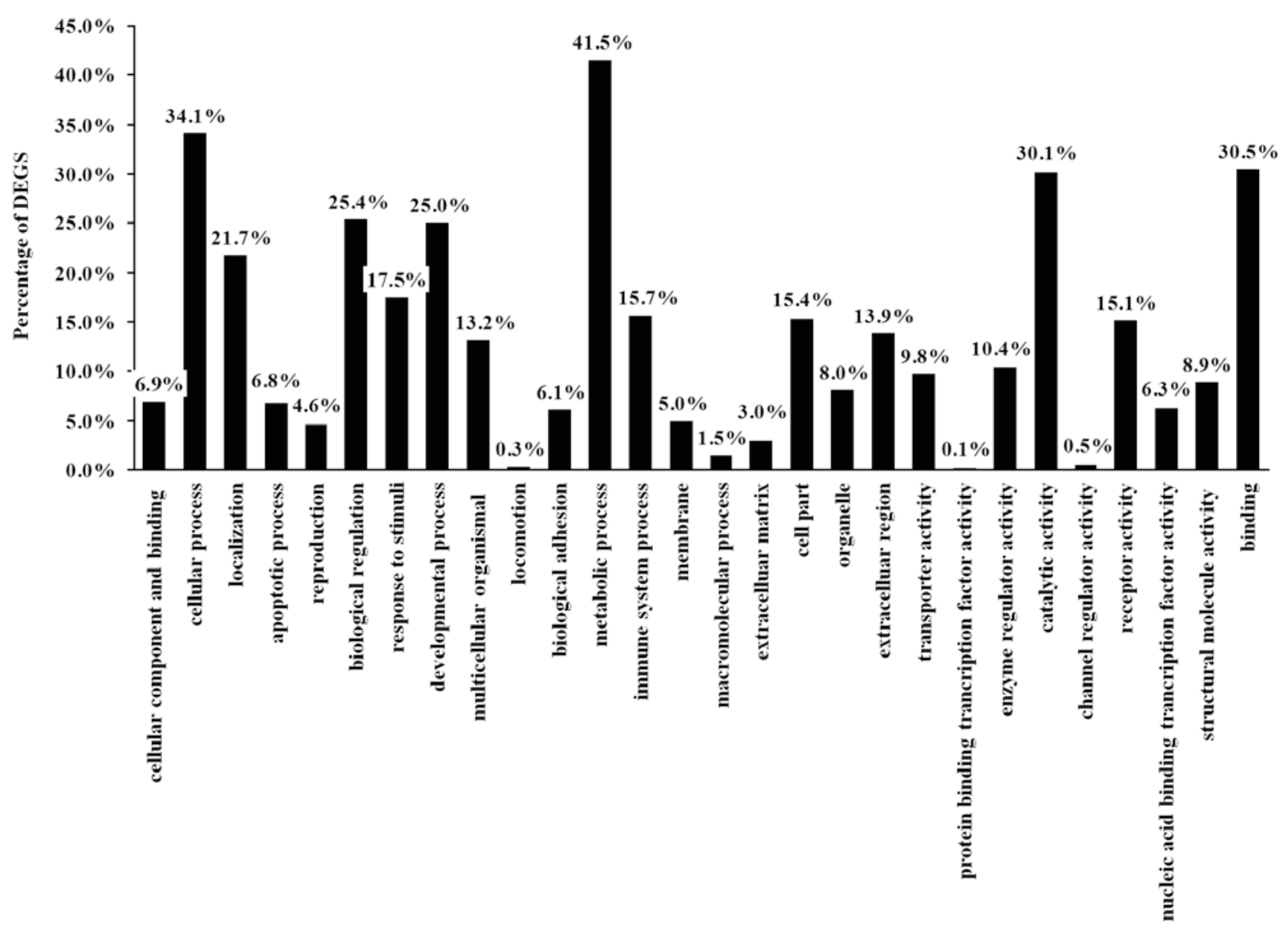

Figure 3. Functional annotation of the significant DEGs. The 236 significant DEGs were categorized by Gene Ontology analysis into 28 functional categories, according to the relevant biological functions of cellular components, molecular functions and biological progresses. Numbers above each column represent the $\%$ of total DEGs involved in each functional category. DEGs, differentially expressed genes. 
apoptosis and organogenesis (22) and has been reported to serve an important role in various human diseases, including cancer. SIX1 is reported to be overexpressed in breast cancer and to promote EMT and metastasis through transforming growth factor (TGF)- $\beta$ signaling (23). SIX1 also acts as a master regulator of the cervical cancer initiation progression; overexpression of SIX1 promotes DNA replication and anchorage-independent growth of cervical cancer cells (24). Furthermore, high expression of SIX1 in cervical cancers enhances vascular endothelial growth factor $\mathrm{C}$ expression by inhibiting TGF- $\beta$ signaling, thus promoting lymphangiogenesis and lymph node metastasis (25). The RNA-Seq data demonstrated that SIX1 was significantly overexpressed in cervical cancer samples with an average $\log _{2}$ change 6.478514 $[\mathrm{P}<0.05$; the reads per kilobase per million mapped reads data of the tumor and normal samples were compared using the empirical Bayes hierarchical model (26)]. Taken together, gene expression patterns in the present study were highly consistent with previous studies indicating that the RNA-Seq data was valid.

In the present study, numerous novel DEGs were also identified in cervical cancer. GBX2 is a homeobox gene that is overexpressed in prostate cancer (27). Overexpression of GBX2 stimulates expression of interleukin (IL)-6 at the transcription level, through binding to an ATTA motif within the promoter of the IL- 6 gene, to promote malignant growth of prostate cancer cells (28). However, the function of GBX2 in other types of cancers has yet to be studied. The results of the present study revealed that GBX2 was significantly upregulated in cervical cancer samples compared with normal tissues. Further studies are required to illuminate the role of GBX2 in cervical cancer. HTR1D belongs to the serotonin receptor family. Knockdown of HTR1D expression in pancreatic cancer cells by small interfering RNAs inhibits cell proliferation and invasion (29). In addition, inhibition of HTR1D suppresses the activity of urokinase plasminogen activator receptor/MMP-2 signaling and integrin/Src/protein tyrosine kinase 2-mediated signaling, in addition to EMT master regulators zinc finger E-box-binding homeobox 1 and Snail family transcriptional repressor 1 (29). EDN signaling serves an important role in cell differentiation, proliferation and migration. EDN signaling is also involved in carcinogenesis, through regulating cell survival and invasiveness (30). Epigenetic activation of EDN-3 through hypermethylation of the EDN3 promoter has been reported in human colon and breast cancer, indicating that EDN3 is a tumor suppressor $(31,32)$. SOSTDC1 has been reported to be downregulated in thyroid cancer cells, breast cancer and in adult and pediatric renal tumors (33-35). SOSTDC1 is a critical regulator of extracellular matrix, through modulating Wnt family member 3A, bone morphogenetic protein (BMP)-2 and BMP-7 signaling in breast cancer cells (34). Serine proteases of the kallikrein family are implicated in various human diseases, including cancer. The expression of KLK family members varies in different types of cancer. Higher expression of KLK13 in breast cancer is associated with improved prognosis, indicating that KLK13 may be a tumor suppressor in breast (36). KLK6 is highly expressed in human non-small cell lung cancer and promotes cell growth and proliferation (37). KLK5 and KLK12 are downregulated in human breast cancer and higher KLK5 and KLK12 expression are associated with improved prognosis $(38,39)$. The data from the present study revealed that GBX2 and HTR1D were overexpressed in cervical cancer tissues, while EDN3, SOSTDC1, KLK5, KLK6, KLK12 and KLK13 were downregulated in cervical cancer tissues, compared with normal tissues. Further functional studies will need to be performed to elucidate the role of these novel DEGs in cervical cancer.

In conclusion, the present study provided a comprehensive transcriptome landscape of cervical cancer and identified novel DEGs in cervical cancer tissues compared with matched normal tissues. These novel genes may be useful for improved understanding of the molecular mechanisms of cervical cancer pathogenesis and potential identification of novel biomarkers and therapeutic targets in the future.

\section{Acknowledgements}

This work was supported by the Fujian Province Science and Technology Plan Key Project (grant no. 2013Y0030) and the National Clinical Key Specialty Construction Program of China.

\section{References}

1. Global Burden of Disease Cancer Collaboration; Fitzmaurice C Dicker D, Pain A, Hamavid H, Moradi-Lakeh M, MacIntyre MF, Allen C, Hansen G, Woodbrook R, et al: The global burden of cancer 2013. JAMA Oncol 1: 505-527, 2015.

2. Torre LA, Bray F, Siegel RL, Ferlay J, Lortet-Tieulent J and Jemal A: Global cancer statistics, 2012. CA Cancer J Clin 65: 87-108, 2015

3. Cogliano V, Baan R, Straif K, Grosse Y, Secretan B and El Ghissassi F; WHO international agency for research on cancer: Carcinogenicity of human papillomaviruses. Lancet Oncol 6: 204, 2005.

4. Arteaga CL and Baselga J: Impact of genomics on personalized cancer medicine. Clin Cancer Res 18: 612-618, 2012.

5. Ozsolak F and Milos PM: RNA sequencing: Advances, challenges and opportunities. Nat Rev Genet 12: 87-98, 2011.

6. Wang Z, Gerstein M and Snyder M: RNA-Seq: A revolutionary tool for transcriptomics. Nat Rev Genet 10: 57-63, 2009.

7. Nagalakshmi U, Wang Z, Waern K, Shou C, Raha D, Gerstein M and Snyder M: The transcriptional landscape of the yeast genome defined by RNA sequencing. Science 320: 1344-1349, 2008.

8. Sultan M, Schulz MH, Richard H, Magen A, Klingenhoff A, Scherf M, Seifert M, Borodina T, Soldatov A, Parkhomchuk D, et al: A global view of gene activity and alternative splicing by deep sequencing of the human transcriptome. Science 321: 956-960, 2008.

9. Stephens PJ, Tarpey PS, Davies H, Van Loo P, Greenman C, Wedge DC, Nik-Zainal S, Martin S, Varela I, Bignell GR, et al: The landscape of cancer genes and mutational processes in breast cancer. Nature 486: 400-404, 2012.

10. Wu Y, Wang X, Wu F, Huang R, Xue F, Liang G, Tao M, Cai P and Huang Y: Transcriptome profiling of the cancer, adjacent non-tumor and distant normal tissues from a colorectal cancer patient by deep sequencing. PLoS One 7: e41001, 2012.

11. Livak KJ and Schmittgen TD: Analysis of relative gene expression data using real-time quantitative PCR and the 2(-Delta Delta C(T)) method. Methods 25: 402-408, 2001.

12. Lv L, Jin Y, Zhou Y, Jin J, Ma Z and Ren Z: Deep sequencing of transcriptome profiling of GSTM2 knock-down in swine testis cells. Sci Rep 6: 38254, 2016.

13. Audic S and Claverie JM: The significance of digital gene expression profiles. Genome Res 7: 986-995, 1997.

14. Benjamini Y and Yekutieli D: The control of the false discovery rate in multiple testing under dependency. Ann Statist 29: $1165-1188,2001$.

15. Abdi H: 'Bonferroni and Sidak corrections for multiple comparisons'. Encyclopedia of Measurement and Statistics. Thousand Oaks, CA: Sage, 2007. 
16. Meijer DH, Kane MF, Mehta S, Liu H, Harrington E, Taylor CM Stiles CD and Rowitch DH: Separated at birth? The functional and molecular divergence of OLIG1 and OLIG2. Nat Rev Neurosci 13: 819-831, 2012.

17. Hanahan D and Weinberg RA: Hallmarks of cancer: The next generation. Cell 144: 646-674, 2011.

18. Conesa A, Madrigal P, Tarazona S, Gomez-Cabrero D, Cervera A McPherson A, Szcześniak MW, Gaffney DJ, Elo LL, Zhang X and Mortazavi A: A survey of best practices for RNA-seq data analysis. Genome Biol 17: 181, 2016.

19. Rajkumar T, Sabitha K, Vijayalakshmi N, Shirley S, Bose MV, Gopal G and Selvaluxmy G: Identification and validation of genes involved in cervical tumourigenesis. BMC Cancer 11: 80 , 2011.

20. Hagemann T, Bozanovic T, Hooper S, Ljubic A, Slettenaar VI, Wilson JL, Singh N, Gayther SA, Shepherd JH and Van Trappen PO: Molecular profiling of cervical cancer progression. Br J Cancer 96: 321-328, 2007.

21. Radisky DC, Levy DD, Littlepage LE, Liu H, Nelson CM, Fata JE, Leake D, Godden EL, Albertson DG, Nieto MA, et al: Raclb and reactive oxygen species mediate MMP-3-induced EMT and genomic instability. Nature 436: 123-127, 2005.

22. Wu W, Ren Z, Li P, Yu D, Chen J, Huang R and Liu H: Six1: A critical transcription factor in tumorigenesis. Int J Cancer 136: $1245-1253,2015$

23. Micalizzi DS, Christensen KL, Jedlicka P, Coletta RD, Barón AE, Harrell JC, Horwitz KB, Billheimer D, Heichman KA, Welm AL, et al: The Six1 homeoprotein induces human mammary carcinoma cells to undergo epithelial-mesenchymal transition and metastasis in mice through increasing TGF-beta signaling. J Clin Invest 119: 2678-2690, 2009.

24. Liu D, Zhang XX, Xi BX, Wan DY, Li L, Zhou J, Wang W, Ma D, Wang $\mathrm{H}$ and Gao QL: Sine oculis homeobox homolog 1 promotes DNA replication and cell proliferation in cervical cancer. Int J Oncol 45: 1232-1240, 2014

25. Liu D, Li L, Zhang XX, Wan DY, Xi BX, Hu Z, Ding WC, Zhu D, Wang XL, Wang W, et al: SIX1 promotes tumor lymphangiogenesis by coordinating TGF $\beta$ signals that increase expression of VEGF-C. Cancer Res 74: 5597-5607, 2014

26. Leng N, Dawson JA, Thomson JA, Ruotti V, Rissman AI, Smits BM, Haag JD, Gould MN, Stewart RM and Kendziorski C: EBSeq: An empirical Bayes hierarchical model for inference in RNA-seq experiments. Bioinformatics 29: 1035-1043, 2013.

27. Gao AC and Isaacs JT: Expression of homeobox gene-GBX2 in human prostatic cancer cells. Prostate 29: 395-398, 1996.

28. Gao AC, Lou W and Isaacs JT: Enhanced GBX2 expression stimulates growth of human prostate cancer cells via transcriptional up-regulation of the interleukin 6 gene. Clin Cancer Res 6 : 493-497, 2000
29. Gurbuz N,Ashour AA, Alpay SN and Ozpolat B: Down-regulation of 5-HT1B and 5-HT1D receptors inhibits proliferation, clonogenicity and invasion of human pancreatic cancer cells. PLoS One 9: e110067, 2014

30. Kawanabe Y and Nauli SM: Endothelin. Cell Mol Life Sci 68 : 195-203, 2011.

31. Wiesmann F, Veeck J, Galm O, Hartmann A, Esteller M, Knüchel R and Dahl E: Frequent loss of endothelin-3 (EDN3) expression due to epigenetic inactivation in human breast cancer. Breast Cancer Res 11: R34, 2009.

32. Wang R, Löhr CV, Fischer K, Dashwood WM, Greenwood JA, Ho E, Williams DE, Ashktorab H, Dashwood MR and Dashwood RH: Epigenetic inactivation of endothelin-2 and endothelin-3 in colon cancer. Int J Cancer 132: 1004-1012, 2013.

33. Liang W, Guan H, He X, Ke W, Xu L, Liu L, Xiao H and Li Y: Down-regulation of SOSTDC1 promotes thyroid cancer cell proliferation via regulating cyclin A2 and cyclin E2. Oncotarget 6: 31780-31791, 2015

34. Clausen KA, Blish KR, Birse CE, Triplette MA, Kute TE, Russell GB, D'Agostino RB Jr, Miller LD, Torti FM and Torti SV: SOSTDC1 differentially modulates Smad and beta-catenin activation and is down-regulated in breast cancer. Breast Cancer Res Treat 129: 737-746, 2011.

35. Blish KR, Clausen KA, Hawkins GA, Garvin AJ, Willingham MC, Turner JC, Torti FM and Torti SV: Loss of heterozygosity and SOSTDC1 in adult and pediatric renal tumors. J Exp Clin Cancer Res 29: 147, 2010.

36. Chang A, Yousef GM, Scorilas A, Grass L, Sismondi P, Ponzone R and Diamandis EP: Human kallikrein gene 13 (KLK13) expression by quantitative RT-PCR: An independent indicator of favourable prognosis in breast cancer. Br J Cancer 86: 1457-1464, 2002.

37. Nathalie HV, Chris P, Serge G, Catherine C, Benjamin B, Claire B, Christelle P, Briollais L, Pascale R, Marie-Lise J and Yves C: High kallikrein-related peptidase 6 in non-small cell lung cancer cells: An indicator of tumour proliferation and poor prognosis. J Cell Mol Med 13: 4014-4022, 2009.

38. Avgeris M, Papachristopoulou G, Polychronis A and Scorilas A Down-regulation of kallikrein-related peptidase 5 (KLK5) expression in breast cancer patients: A biomarker for the differential diagnosis of breast lesions. Clin Proteomics 8: 5, 2011.

39. Talieri M, Devetzi M, Scorilas A, Pappa E, Tsapralis N, Missitzis I and Ardavanis A: Human kallikrein-related peptidase 12 (KLK12) splice variants expression in breast cancer and their clinical impact. Tumour Biol 33: 1075-1084, 2012. 